\title{
Etude de la viscosité des " rétentats" et des "préfromages " obtenus après traitement du lait par ultrafiltration sur membrane
}

\author{
par \\ J. CULIOLI, J.P. BON et J. L. MAUBOIS \\ avec la collaboration technique de H. GOUDEDRANCHE et M. PIOT \\ Laboratoire de Recherches de Technologie Laitière, I.N.R.A., Rennes*
}

L'application de l'ultrafiltration sur membrane au traitement du lait a permis d'élaborer une technique nouvelle de fabrication du fromage (Maubois et al., 1969 ; Maubois et Mocquot, 1971).

La proportion des différents composants et la concentration en substance sèche du liquide qui ne passe pas au travers de la membrane, le "rétentat ", varient au cours de l'ultrafiltration. Il se produit une concentration sélective des protéines du lait et des sels minéraux qui leur sont liés (phosphate de calcium associé aux micelles de caséine).

Cette élévation de la teneur en protéines du " rétentat » modifie sensiblement ses propriétés rhéologiques et notamment sa viscosité.

Différents auteurs ont mis en évidence l'influence de la composition du lait et de ses dérivés (Cox, 1959), de la température, du $\mathrm{pH}$ et de l'état physique des phases dispersées, micelles de caséine et globules gras (Beeby et Kumetat, 1959 ; Beeby et Lee, 1959), sur les propriétés rhéologiques de ces produits. L'influence de divers traitements technologiques tels que l'action de la chaleur, l'agitation, l'homogénéisation a également été étudiée (Prentice et Chapman, 1969). A partir des calculs de régression effectués sur un grand nombre de résultats expérimentaux, Fernandez-Martin (1972) a établi une expression mathématique (fonction polynomiale) reliant le logarithme de la viscosité du lait à la température et à la concentration.

Mais ces résultats ne s'appliquent pas aux " rétentats » d'ultrafiltration de lait car un lait concentré par évaporation se trouve enrichi en tous ses constituants alors qu'un " rétentat » d'ultrafiltration du lait est enrichi essentiellement en protéines. Or la

* Adresse : 65, rue de Saint-Brieuc - 35042 Rennes cedex. 
viscosité du lait est principalement fonction de sa teneur en protéines et en matières grasses (Whitnah, 1962).

La connaissance des propriétés rhéologiques des "rétentats" et des produits obtenus par addition de crème aux « rétentats " ( " préfromages ») est particulièrement utile parce que ces propriétés déterminent non seulement le choix des températures de mise en œuvre de l'ultrafiltration (Maubois, 1973) mais aussi les caractéristiques hydrodynamiques optimales des appareillages composant une installation d'ultrafiltration.

En effet, l'élévation de la teneur en protéines par ultrafiltration accroît la viscosité du "rétentat " et accélère la formation d'une couche de polarisation de concentration à la surface des membranes (Blatt et al., 1970 ; Merson et Ginnette, 1972). Les équations établies par Blatt et al. (1970) et Merson et Ginnette (1972) pour représenter mathématiquement ce phénomène montrent que l'épaisseur de cette couche est directement proportionnelle à la viscosité du "rétentat ". Outre la diminution des débits d'ultrafiltration, la polarisation de concentration peut provoquer dans certaines conditions une rétention de sels minéraux et de lactose incompatible avec l'obtention ultérieure de fromages de bonne qualité (Maubois, 1973).

La connaissance de l'évolution des propriétés rhéologiques des "rétentats " d'ultrafiltration en fonction de divers paramètres (température, gradient de vitesse, etc.) permet de choisir les conditions de mise en ouvre de l'ultrafiltration propres à réduire, autant que faire se peut, ce phénomène de polarisation de concentration.

En outre, les pompes, les tuyauteries, les échangeurs de température, les dispositifs d'agitation et de mélange, les appareillages de dosage et de moulage du "préfromage liquide », doivent être adaptés à la viscosité de chaque produit.

Nous nous sommes donc attachés au cours du présent travail à étudier non seulement la variation de la viscosité des "rétentats " d'ultrafiltration et des « préfromages liquides » en fonction de leur composition mais aussi l'influence que pouvaient avoir sur cette viscosité différents paramètres physico-chimiques.

\section{MATERIEL ET METHODES}

\section{1) Obtention des produits étudiés}

- Lait : Le lait utilisé était du lait écrémé de grand mélange ayant subi une pasteurisation H.T.S.T. $\left(72^{\circ} \mathrm{C}-15 \mathrm{~s}\right)$.

- Laits concentrés : Les échantillons de lait concentré à différentes teneurs en substance sèche étaient obtenus par dilution progressive avec de l'eau distillée, à température ambiante, d'un lait 
concentré non sucré préparé à l'échelle industrielle et contenant 49 p. 100 de substance sèche.

- "Rétentats » d'ultrafiltration : Les ultrafiltrations étaient réalisées suivant le processus indiqué par Maubois (1973). Le lait écrémé était ultrafiltré soit directement, soit après inoculation d'un levain sur lait de bactéries lactiques mésophiles à la dose de 1 p. 100 et «maturation " du lait inoculé à la température de $28^{\circ} \mathrm{C}$ jusqu'à pH 6,0.

Deux appareillages étaient utilisés :

- un module Rhône-Poulenc type UFP 20, équipé de membranes Iris 3042 représentant une surface effective filtrante de $11 \mathrm{~m}^{2}$,

- un module D.D.S. (De Danske Sukkerfabrikker) équipé de membranes 600 représentant une surface effective filtrante de $18 \mathrm{~m}^{2}$.

L'ultrafiltration était réalisée à basse température $\left(2^{\circ} \mathrm{C}\right.$ à $\left.4^{\circ} \mathrm{C}\right)$ jusqu'à l'obtention d'un "rétentat » ayant une teneur en matières azotées totales (M.A.T.) de $10 \mathrm{~g} \mathrm{p} .100 \mathrm{~g}$ de "rétentat », puis à $30^{\circ} \mathrm{C}$ jusqu'à l'obtention du « rétentat » final ayant une teneur en M.A.T. voisine de $18 \mathrm{~g} \mathrm{p} .100 \mathrm{~g}$ de « rétentat ». Des échantillons de « rétentat » étaient prélevés à différents stades de l'ultrafiltration. Dans quelques essais, ces échantillons étaient immédiatement additionnés de 0,2 $\mathrm{ml}$ d'eau oxygénée (à 30 p. 100 en poids) pour $100 \mathrm{ml}$ de produit, afin d'arrêter la croissance des bactéries lactiques (Kerhervé et Richard, 1971), provenant de l'inoculation du lait (soumis à l'ultrafiltration) par un levain lactique.

— «Préfromages liquides »: Les «préfromages " destinés à la fabrication de fromage à " pâte fraîche » étaient obtenus en ajoutant aux « rétentats » correspondants $(7,4 \mathrm{~g}$ de M.A.T. p. $100 \mathrm{~g}$ de « rétentat »), à température ambiante, une quantité convenable de crème (40 p. 100 de matière grasse) pour obtenir un rapport matière grasse/substance sèche voisin de 0,4 .

Les «préfromages 》 destinés à la fabrication des fromages à " pâte molle " étaient obtenus en ajoutant aux « rétentats » correspondants (18 g environ de M.A.T. p. $100 \mathrm{~g}$ de "rétentat ») de la crème ( 50 p. 100 de matière grasse), sous agitation lente à $30^{\circ} \mathrm{C}$, en quantité suffisante pour que le rapport matière grasse/matière sèche du mélange soit égal ou voisin de 0,4 .

Chaque échantillon était divisé en fractions aliquotes sur lesquelles on effectuait les analyses et les mesures de viscosité.

\section{2) Déterminations analytiques}

- Les teneurs en substance sèche (E.S.T.) étaient déterminées par dessiccation à l'étuve $\left(102^{\circ} \mathrm{C}-104^{\circ} \mathrm{C}\right)$ pendant $7 \mathrm{~h}$, d'environ $5 \mathrm{~g}$ de produit.

- Les teneurs en matières azotées totales (M.A.T.) étaient obtenues en multipliant la teneur en azote total, déterminée par microkjeldahl, par le coefficient 6,38 . 
- Les teneurs en matière grasse (M.G.) de la crème et des "préfromages » étaient déterminées après extraction par le mélange éther de pétrole-éther diéthylique (Norme de la Fédération Internationale de Laiterie - 5 - 1959).

\section{3) Mesures de viscosité}

Les mesures de viscosité étaient réalisées par détermination directe des tensions de cisaillement, pour des gradients de vitesse définis, à l'aide d'un viscosimètre rotatif, type Couette, à cylindres coaxiaux (Rhéotest II). Cet appareil convient non seulement pour la détermination de la viscosité dynamique des liquides newtoniens (1), mais aussi pour l'étude des caractéristiques rhéologiques des liquides non newtoniens (2). La température des échantillons était maintenue à $\pm 0,2^{\circ} \mathrm{C}$ à l'aide d'un thermostat à circulation d'eau.

\section{RESULTATS}

\section{1) Viscosité du lait écrémé}

Les courbes représentées dans la figure 1 indiquent la variation de viscosité, exprimée en centipoises (cP), du lait écrémé de grand mélange utilisé dans nos essais, en fonction de la valeur, exprimée en $\mathrm{s}^{-1}$, du gradient de vitesse, à $2^{\circ} \mathrm{C}$ et à $30^{\circ} \mathrm{C}$.

Pour des valeurs du gradient de vitesse comprises entre 0 et $400 \mathrm{~s}^{-1}$, les tensions de cisaillement, dans le cas du lait écrémé, sont trop faibles (inférieures à $10 \mathrm{dyn} / \mathrm{cm}^{2}$ ) pour être mesurées avec précision sur le viscosimètre, du type Couette, Rhéotest II.

Pour des valeurs du gradient de vitesse supérieures à $400 \mathrm{~s}^{-1}$, le lait écrémé présente un comportement newtonien. La viscosité, dans ce cas, est, pour une température donnée, pratiquement indépendante du gradient de vitesse. Elle est égale à 2,0 centipoises $(\mathrm{cP})$ à la température de $30^{\circ} \mathrm{C}$ et 3,3 centipoises à la température de $2^{\circ} \mathrm{C}$.

(1) Un liquide est dit newtonien lorsque le coefficient de viscosité ( $\eta$ ) de l'équation de Newton $\left(F=\eta n . S . \frac{d v}{d x}\right)$ est constant quelle que soit la valeur du gradient de vitesse $(D=d v / d x)$ et de la tension de cisaillement $(\tau=F / S)$.

(2) Un liquide est dit non newtonien si sa viscosité est fonction de la tension de cisaillement ou du gradient de vitesse agissant sur ce liquide. Selon la forme des courbes $n=f(D)$ et $\pi=f(D)$ obtenues, les liquides non newtoniens peuvent être caractérisés sur le plan rhéologique comme présentant une viscosité de structure, un état de dilatance, de plasticité ou de pseudo-plasticité ou encore la propriété de thixotropie ou de rhéopexie (Szczeniak, 1973). 
VISCOSITE ( $C P)$

$2^{\circ} \mathrm{C}$

$30^{\circ} \mathrm{C}$

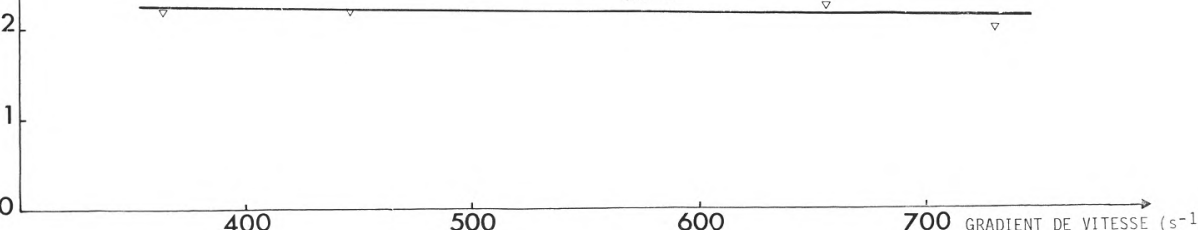

fig. 1

Influence du gradient de vitesse sur la viscosité d'un lait écrémé de grand mélange :

O température $2^{\circ} \mathrm{C}$

$\nabla$ température $30^{\circ} \mathrm{C}$

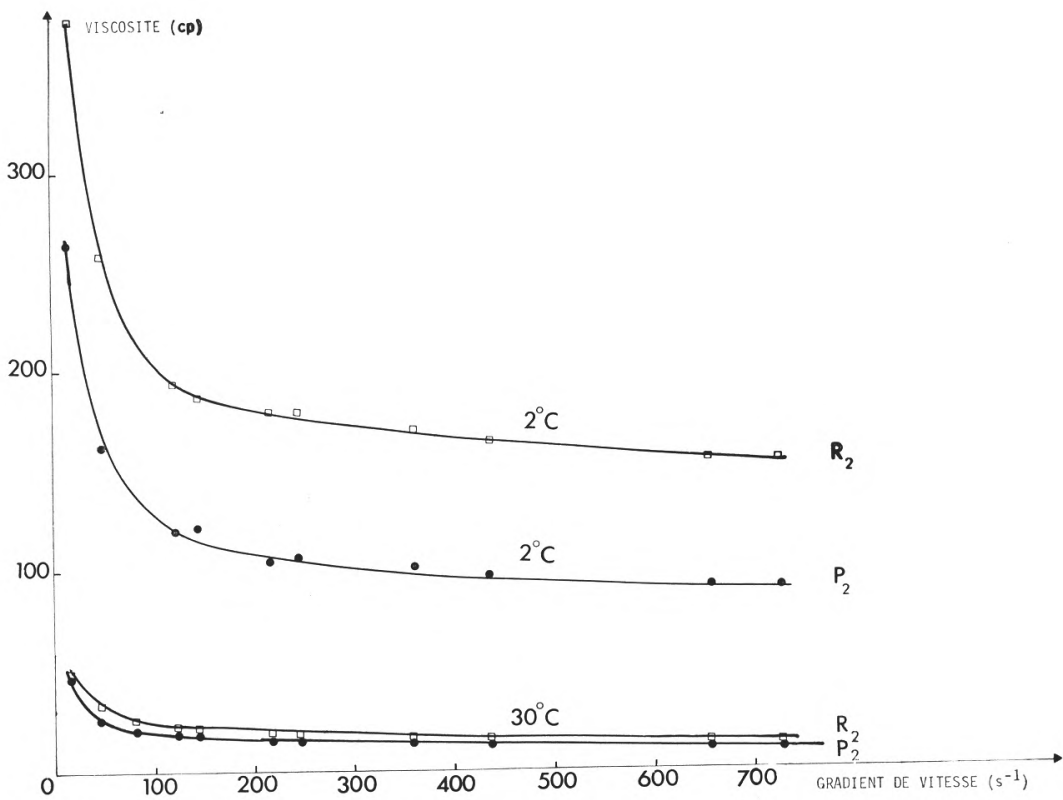

fig. 2

Influence du gradient de vitesse sur la viscosité d'un " rétentat » et d'un «préfromage » du type "pâte molle » :

rétentat

- préfromage 


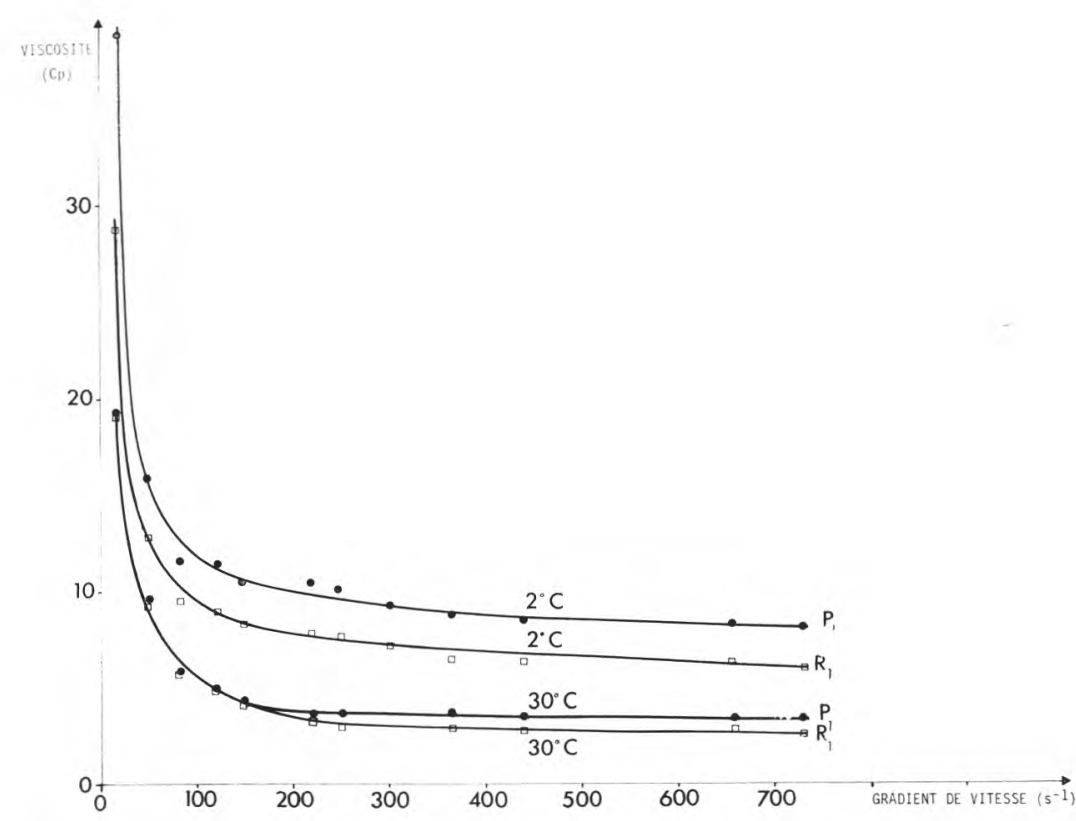

fig. 3

Influence du gradient de vitesse sur la viscosité d'un " rétentat » et d'un "préfromage " du type "pâte fraîche ":

c rétentat

- préfromage

\section{2) Viscosité des " rétentats " d'ultrafiltration sur membrane}

\section{a) Influence du gradient de vitesse}

Les " rétentats " d'ultrafiltration ne présentent pas un comportement rhéologique newtonien pour les faibles valeurs du gradient de vitesse (valeurs inférieures à $300 \mathrm{~s}^{-1}$ ). En effet, l'examen des courbes Re de la figure 2 montre que la viscosité des " rétentats » d'ultrafiltration dont la teneur en M.A.T. est de 17,5 g p. $100 \mathrm{~g}$ de "rétentat » est très dépendante de la valeur du gradient de vitesse. Cette diminution de la viscosité en fonction de l'accroissement du gradient de vitesse, caractéristique d'une viscosité de structure, est plus marquée à $2^{\circ} \mathrm{C}$ qu'à $30^{\circ} \mathrm{C}$. Entre les gradients de vitesse $16,2 \mathrm{~s}^{-1}$ et $243 \mathrm{~s}^{-1}$, la chute de viscosité est de $30 \mathrm{cP}$ à $30^{\circ} \mathrm{C}$ et de $196 \mathrm{cP}$ à $2^{\circ} \mathrm{C}$.

Lorsque la valeur du gradient de vitesse dépasse $300 \mathrm{~s}^{-1}$, les " rétentats » d'ultrafiltration deviennent des liquides presque newtoniens. 


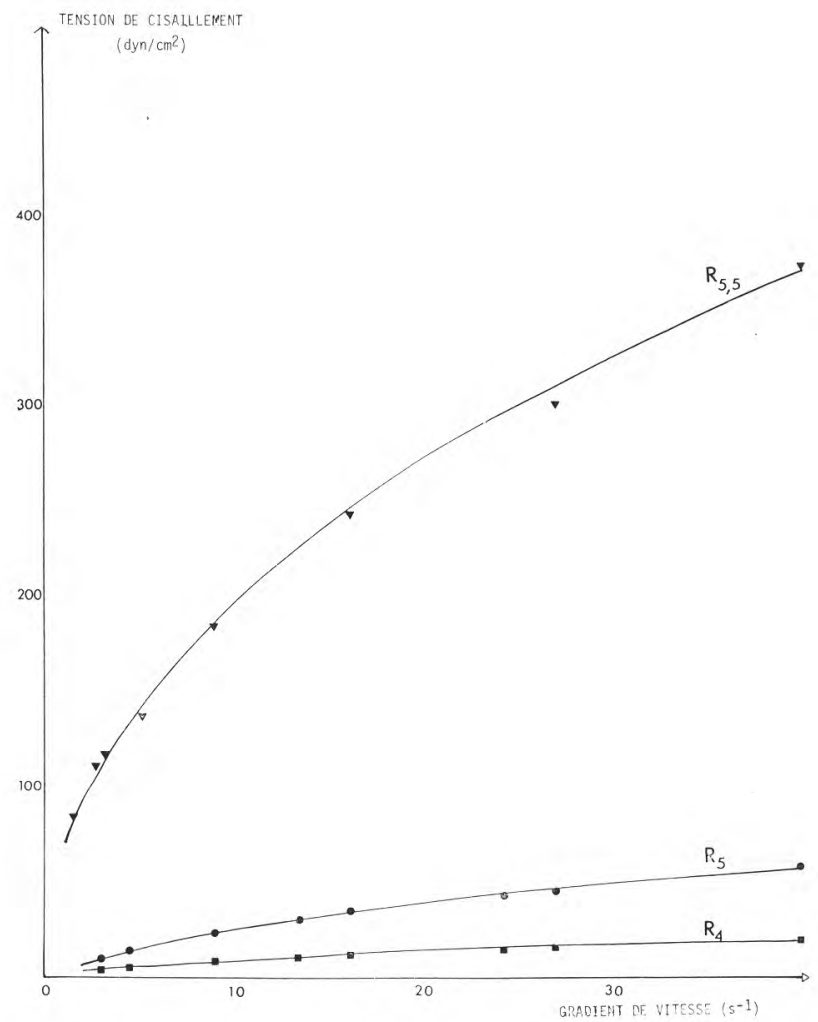

fig. 4

Variation de la tension de cisaillement à $2^{\circ} \mathrm{C}$ en fonction du gradient de vitesse pour des "rétentats » à divers stades de l'ultrafiltration réalisée à $\mathrm{pH}$ 6,6:

Teneur en protéines des « rétentats»:

-12,0 g p. $100 \mathrm{~g}$

- 15,0 g p. $100 \mathrm{~g}$

$\nabla 16,9$ g p. $100 \mathrm{~g}$

La figure 4 indique les variations de la tension de cisaillement $(\tau)$ en fonction du gradient de vitesse (D) observées pour des « rétentats » d'ultrafiltration à $\mathrm{pH} 6,6$ contenant respectivement $12,0 \mathrm{~g}\left(\mathrm{R}_{4}\right), 15,0 \mathrm{~g}$ $\left(R_{5}\right)$ et $16,9 \mathrm{~g}\left(\mathrm{R}_{5,5}\right)$ de M.A.T. pour $100 \mathrm{~g}$ de « rétentat».

Dans le domaine de gradient de vitesse étudié 0 à $40 \mathrm{~s}^{-1}$, le rapport $\mathrm{d} \tau / \mathrm{dD}$ qui représente la viscosité est continuellement décroissant. De telles courbes sont caractéristiques d'un comportement pseudoplastique (Szczesniak, 1973). Sur la figure 4, on peut également remarquer un très fort accroissement de la tension de cisaillement lorsque la teneur en M.A.T. du "rétentat " d'ultrafiltration passe de 15,0 g à $16,9 \mathrm{~g} \mathrm{p} .100 \mathrm{~g}$. 


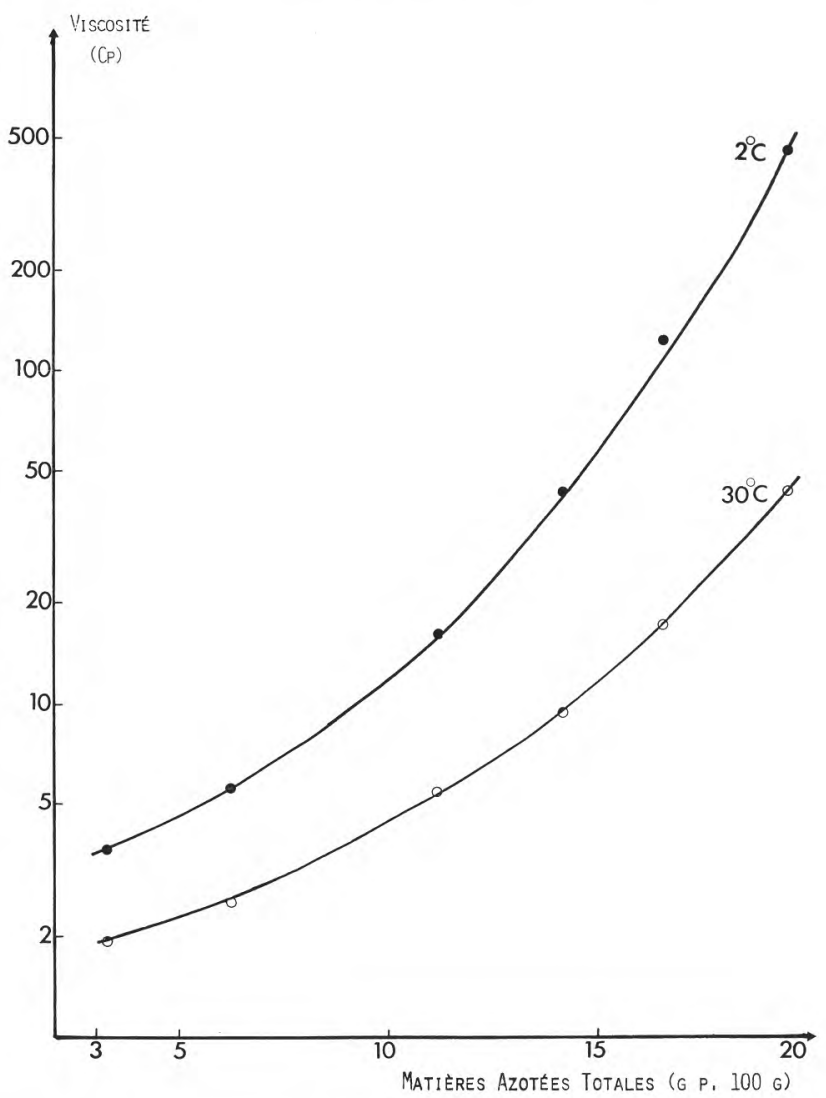

fig. 5

Variation de la viscosité du "rétentat " au cours de l'ultrafiltration, à $\mathrm{pH}$ 6,6, d'un lait écrémé de grand mélange :

- température $2^{\circ} \mathrm{C}$

O température $30^{\circ} \mathrm{C}$

Gradient de vitesse : $437,4 \mathrm{~s}^{-1}$

b) Influence de la teneur en M.A.T. et de la teneur en substance sèche

Nous avons étudié l'influence du traitement du lait par ultrafiltration sur la viscosité, à deux températures (2० C et $30^{\circ} \mathrm{C}$ ) et à un gradient de vitesse $\left(437,4 \mathrm{~s}^{-1}\right)$ pour lequel les " rétentats » d'ultrafiltration présentent un comportement quasi-newtonien. Les résultats obtenus sont indiqués dans la figure 5 .

L'augmentation de la teneur en M.A.T. provoque un accroissement très important de la viscosité qui varie de $2,0 \mathrm{cP}$ à 4,5 cP à la température de $30^{\circ} \mathrm{C}$ et de $3,6 \mathrm{cP}$ à $454 \mathrm{cP}$ à la température de $2^{\circ} \mathrm{C}$, 


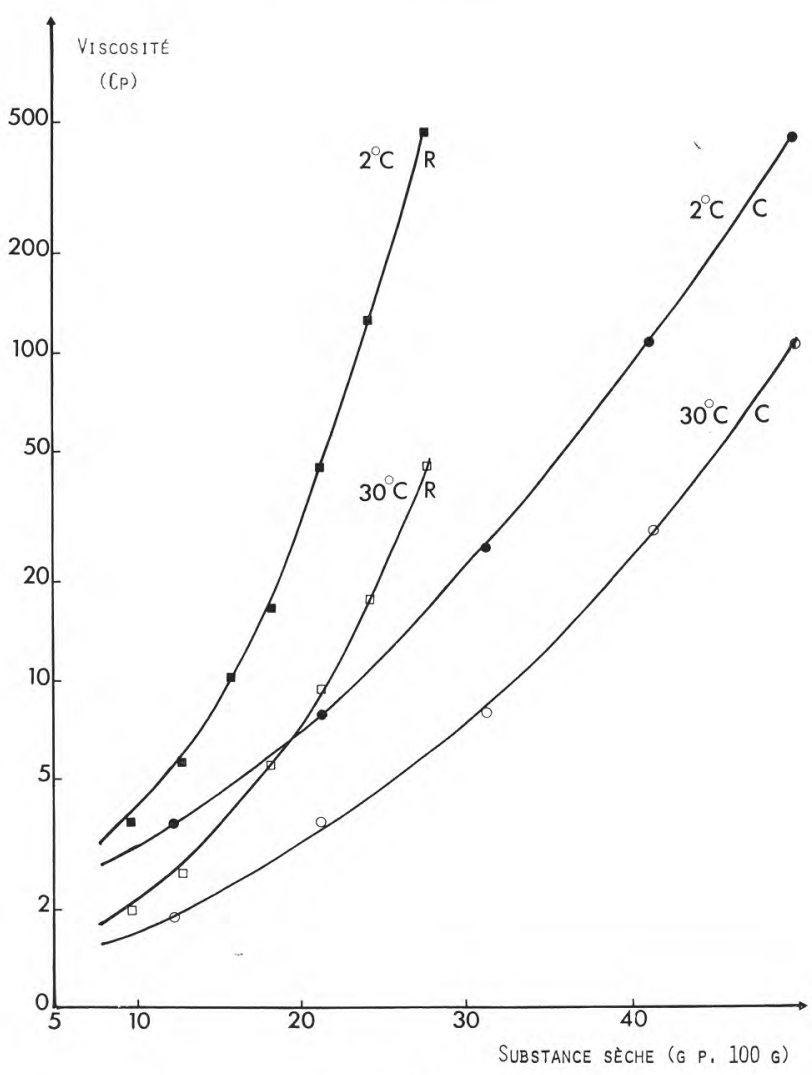

fig. 6

Influence du facteur de concentration sur la viscosité du « rétentat » d'ultrafiltration à $\mathrm{pH}$ 6,6 et du lait concentré par évaporation :

Rétentat

Lait concentré par évaporation

Gradient de vitesse : $437,4 \mathrm{~s}^{-1}$
- $2^{\circ} \mathrm{C}$

- $2^{\circ} \mathrm{C}$,

ㅁ $30^{\circ} \mathrm{C}$

○ $30^{\circ} \mathrm{C}$

pour des teneurs en M.A.T. allant respectivement de 3,3 g p. $100 \mathrm{~g}$ à $19,6 \mathrm{~g} \mathrm{p} .100 \mathrm{~g}$.

Dans le but d'étudier l'influence, sur la viscosité, de la proportion relative des différents constituants du lait, nous avons indiqué, sur la figure 6 , les variations de la viscosité aux températures de $2^{\circ} \mathrm{C}$ et de $30^{\circ} \mathrm{C}$, en fonction de la teneur en substance sèche, à la fois pour les "rétentats » d'ultrafiltration et pour les laits concentrés par évaporation.

Pour une même teneur en substance sèche, la viscosité des " rétentats » est plus élevée que celle des laits concentrés. L'écart 
entre la viscosité des deux produits est d'autant plus important que la teneur en substance sèche est plus élevée. A $2^{\circ} \mathrm{C}$, cet écart, égal à $23 \mathrm{cP}$ pour une teneur en substance sèche de $20 \mathrm{~g} \mathrm{p} .100 \mathrm{~g}$, atteint $150 \mathrm{cP}$ pour une teneur en substance sèche de $25 \mathrm{~g} \mathrm{p} .100 \mathrm{~g}$. A $30^{\circ} \mathrm{C}$, la différence de viscosité croît moins fortement ; elle passe de 4,3 à $12 \mathrm{cP}$ pour la même variation de teneur en substance sèche.

\section{c) Influence $d u p H$}

Dans la technique décrite par Maubois (1973), le $\mathrm{pH}$ du lait est abaissé avant ultrafiltration à la valeur 6,0 par inoculation d'un levain lactique et incubation à une température comprise entre $28^{\circ} \mathrm{C}$ $30^{\circ} \mathrm{C}$. L'ultrafiltration est ensuite réalisée d'abord à $2^{\circ} \mathrm{C}$ (température à laquelle le $\mathrm{pH}$ du « rétentat » ne varie pas), puis à $30^{\circ} \mathrm{C}$. A cette dernière température, le $\mathrm{pH}$ du "rétentat " s'abaisse progressivement de 6,0 à 5,4 par suite du développement des bactéries lactiques (tab. 1).

TABLEAU 1

Composition et $\mathrm{pH}$ des " rétentats » au cours d'une opération d'ultrafiltration*

\begin{tabular}{c|c|c|c}
\hline Echantillon & $\mathrm{pH}$ & E.S.T.** & M.A.T.** \\
\hline & & & \\
\hline 1 & 6,0 & 9,0 & 3,5 \\
2 & 6,0 & 13,5 & 7,3 \\
3 & 6,0 & 15,5 & 9,2 \\
4 & 5,7 & 19,3 & 12,8 \\
5 & 5,6 & 21,3 & 15,1 \\
6 & 5,4 & 24,5 & 17,6 \\
& & & \\
\hline
\end{tabular}

* Voir dans le texte le détail des conditions d'opération.

** Résultats exprimés en g de substance sèche totale (E.S.T.) ou de matières azotées totales (M.A.T.) pour $100 \mathrm{~g}$ de « rétentat».

L'influence sur la viscosité des " rétentats " obtenus est la suivante (fig. 7) : les courbes indiquent les variations, à $2^{\circ} \mathrm{C}$ et $30^{\circ} \mathrm{C}$, de la viscosité des "rétentats » en fonction de leur teneur en M.A.T., dans le cas d'un lait ultrafiltré à $\mathrm{pH}$ 6,6 et dans le cas d'un lait ultrafiltré à $\mathrm{pH}$ acide $(6,0-5,4)$.

$\mathrm{Si}$ on exprime la variation de la viscosité en fonction du $\mathrm{pH}$ par le rapport $\frac{\eta_{1}-\eta_{2}}{\eta_{1}} \times 100$.

$\eta_{1}$ étant la viscosité d'un « rétentat » à $\mathrm{pH} 6,6$,

$n_{2}$ étant la viscosité du « rétentat " correspondant (teneur en M.A.T. identique) acidifié, l'abaissement de viscosité est de l'ordre 


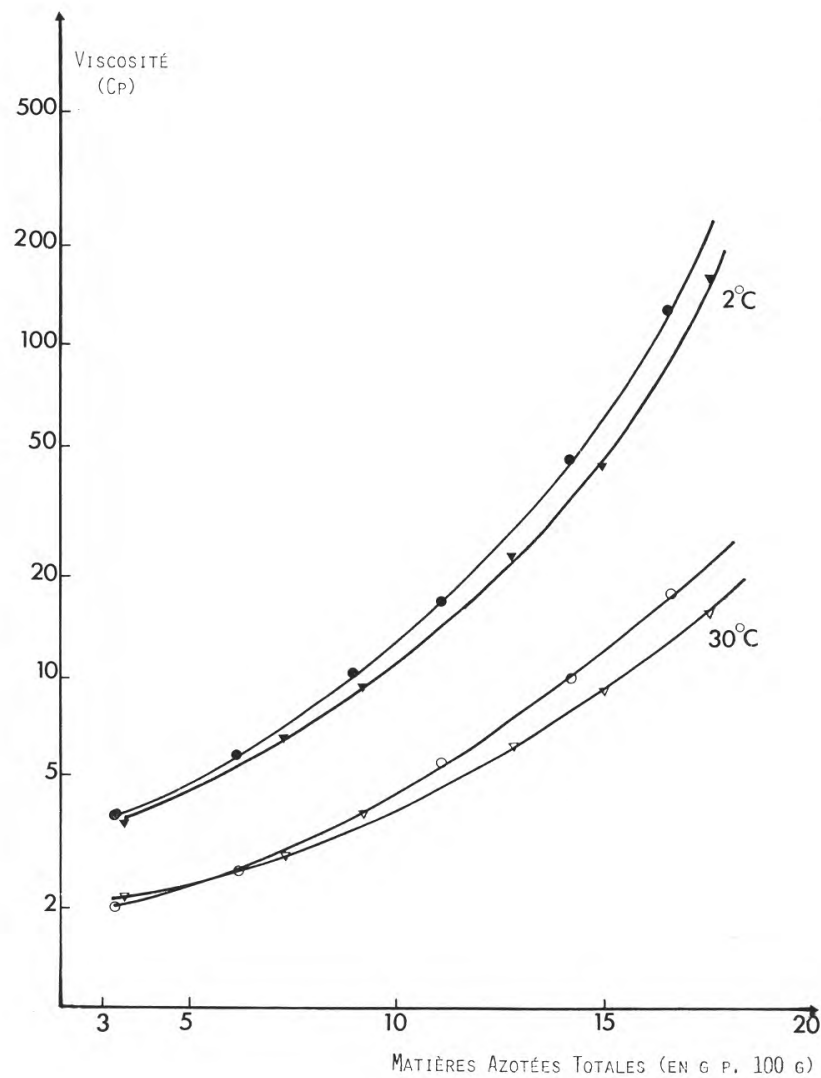

fig. 7

Influence du pH sur la viscosité des "rétentats " d'ultrafiltration d'un lait de grand mélange :

Ultrafiltration à $\mathrm{pH}$ 6,0-5,4

Ultrafiltration à $\mathrm{pH}$ 6,6

Gradient de vitesse : $437,4 \mathrm{~s}^{-1}$

$\nabla 2^{\circ} \mathrm{C}, \quad \nabla 30^{\circ} \mathrm{C}$

- $2^{\circ} \mathrm{C}, \quad \mathrm{O} 30^{\circ} \mathrm{C}$

de 12 p. 100 pour une teneur en M.A.T. égale à $10 \mathrm{~g} \mathrm{p} .100 \mathrm{~g}$ et de l'ordre de 30 p. 100 pour une teneur en M.A.T. égale à $17,5 \mathrm{~g} \mathrm{p} .100 \mathrm{~g}$ à $2^{\circ} \mathrm{C}$ comme à $30^{\circ} \mathrm{C}$.

d) Influence de la conservation au froid

Au cours de cette étude, nous avons utilisé des " rétentats 》 préparés sur module D.D.S. équipé de membranes 600 . L'ultrafiltration était conduite à $\mathrm{pH}$ 6,6, sans fermentation lactique, comme indiqué ci-dessus, pendant $1 \mathrm{~h} 50 \mathrm{mn}$ à la température de $2^{\circ} \mathrm{C}$ et $50 \mathrm{mn}$ à la température de $30^{\circ} \mathrm{C}$. 


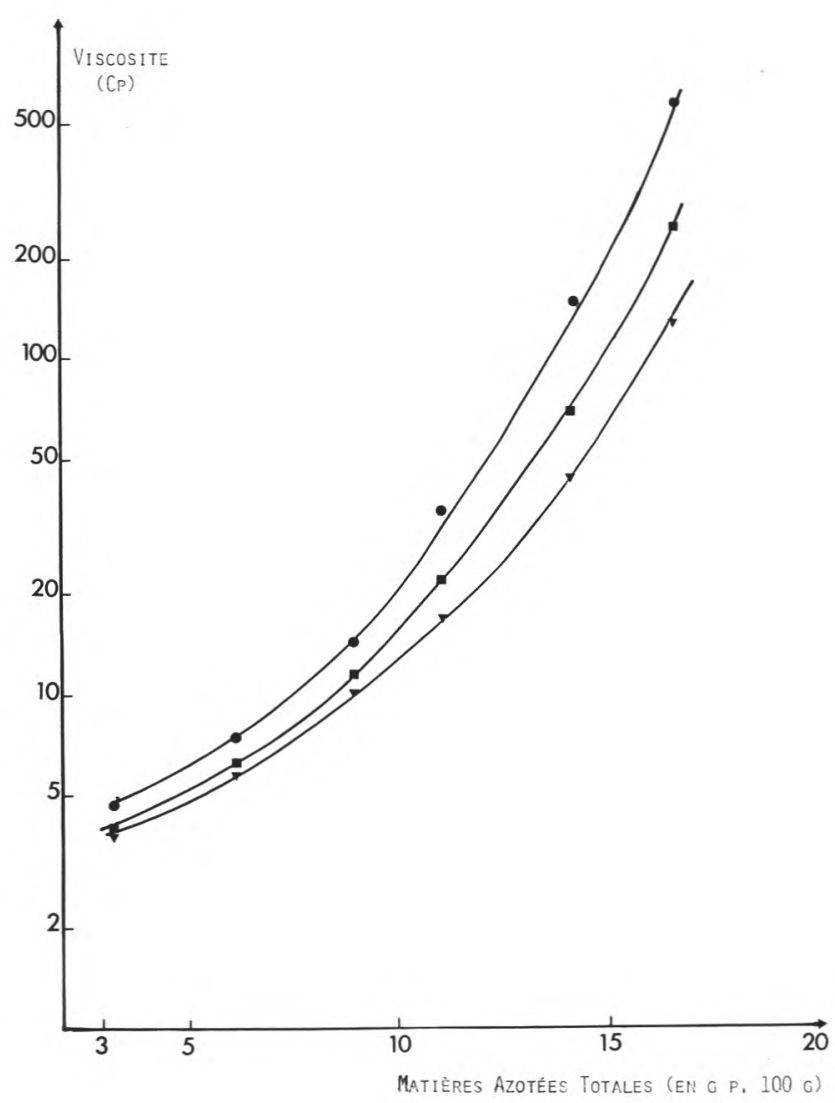

fig. 8

Evolution de la viscosité de "rétentats " d'ultrafiltration d'un lait de grand mélange au cours de la conservation à la température de $2^{\circ} \mathrm{C}$ :

Durée de conservation (en heures)

Gradient de vitesse : $437,4 \mathrm{~s}^{-1}$

$\boldsymbol{\nabla} 0, \quad \mathbf{a} 24, \quad \bullet 96$

Les " rétentats " prélevés aux différents stades de l'ultrafiltration étaient, selon le cas, soit maintenus à $2^{\circ} \mathrm{C}$, soit refroidis progressivement de $30^{\circ} \mathrm{C}$ à $2^{\circ} \mathrm{C}$.

Les mesures de viscosité étaient réalisées à la température de $2^{\circ} \mathrm{C}$, immédiatement, et après $24 \mathrm{~h}$ et $96 \mathrm{~h}$ de conservation à $2^{\circ} \mathrm{C}$.

Les résultats rassemblés dans la figure 8 montrent que la conservation au froid $\left(2^{\circ} \mathrm{C}\right)$ de "rétentats " à $\mathrm{pH} 6,6$ non additionnés d'eau oxygénée, a pour conséquence un accroissement important de leur viscosité. 
Pour des teneurs en M.A.T. égales à 6,2, 11,1 et 16,5 g p. 100 g, la viscosité des "rétentats " à la température de $2^{\circ} \mathrm{C}$, est, après $24 \mathrm{~h}$ de conservation, respectivement multipliée par $1,1,1,3$ et 1,9 et, après 96 h de conservation, par 1,3, 2,1 et 4,7.

Pour des teneurs en protéines supérieures à 16 g p. 100 g, la conservation au froid $\left(+2^{\circ} \mathrm{C}\right)$ provoque une prise en masse progressive du « rétentat » conduisant à la formation d'un gel, ne présentant pas de phénomène de synérèse. Cette gélification est réversible ; la structure de gel est détruite par une élévation de la température.

Dans le cas où les « rétentats » ont été préalablement additionnés d'eau oxygénée dans les conditions décrites ci-dessus, l'accroissement de viscosité au cours de la conservation au froid $\left(+2^{\circ} \mathrm{C}\right)$ est plus important. En outre, pour des teneurs en M.A.T. supérieures à $16 \mathrm{~g}$ p. $100 \mathrm{~g}$, la gélification des " rétentats " se produit plus rapidement.

\section{3) Viscosité des " préfromages liquides"}

Après avoir étudié les propriétés rhéologiques du lait écrémé et des « rétentats » prélevés aux différents stades de l'ultrafiltration sur membrane, nous avons comparé les propriétés d'écoulement de deux "préfromages » $\left(\mathrm{P}_{1}\right.$ et $\left.\mathrm{P}_{2}\right)$ à celles des " rétentats » $\mathrm{R}_{1}$ et $\mathrm{R}_{2}$ dont ils étaient issus. La composition de ces produits est indiquée dans le tableau 2.

\section{TABLEAU 2}

Caractéristiques du lait, de la crème, de "rétentats » d'ultrafiltration $\left(\mathrm{R}_{1}, \mathrm{R}_{2}\right)$ et de "préfromages " $\left(\mathrm{P}_{1}, \mathrm{P}_{2}\right)$

\begin{tabular}{|c|c|c|c|c|}
\hline \multirow{2}{*}{ Echantillons } & \multirow{2}{*}{$\mathrm{pH}$} & \multirow{2}{*}{ E.S.T.* } & \multirow{2}{*}{ M.A.T.* } & M.G.** \\
\hline & & & & E.S.T. \\
\hline Lait écrémé après maturation & 6,0 & 9,0 & 3,4 & - \\
\hline Crème & - & 54,0 & - & 0,92 \\
\hline Rétentat $R_{1}$ & 6,0 & 13,5 & 7,3 & - \\
\hline Rétentat $\mathrm{R}_{2}$ & 5,4 & 24,5 & 17,6 & - \\
\hline Préfromage $P_{1}$ & 6,0 & 21,3 & 6,1 & 0,46 \\
\hline Préfromage $P_{2}$ & 5,4 & 32,2 & 13,1 & 0,42 \\
\hline
\end{tabular}

* Résultats exprimés en g de substance sèche totale (E.S.T.) ou de matières azotées totales (M.A.T.) pour $100 \mathrm{~g}$ de rétentat.

** $\frac{\text { M.G. }}{\text { E.S.T. }}$ Teneur en matière grasse/teneur en substance sèche totale. 


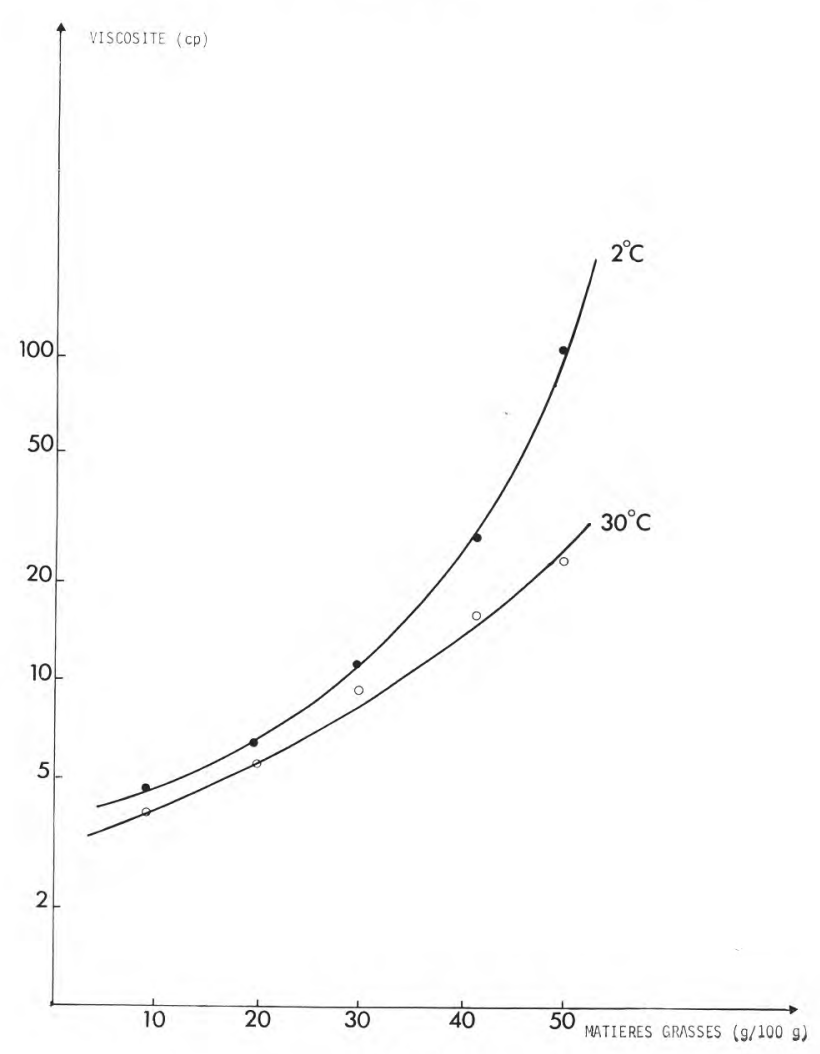

fig. 9

Influence de la teneur en matière grasse sur la viscosité de la crème

- température $2^{\circ} \mathrm{C}$

O température $30^{\circ} \mathrm{C}$

Gradient de vitesse : $437,4 \mathrm{~s}^{-1}$

Le " rétentat " $R_{1}$ avait une teneur en substance sèche identique à celle d'un fromage à pâte fraîche maigre et le « rétentat " $R_{2}$ avait la composition recherchée pour la préparation d'un « préfromage " à pâte molle du type Camembert.

Nous avons reporté sur les figures 2 et 3 la viscosité de ces " rétentats » et de ces «préfromages » en fonction du gradient de vitesse. Les mesures ont été effectuées à $2^{\circ} \mathrm{C}$ et à $30^{\circ} \mathrm{C}$. Les courbes obtenues indiquent que le comportement rhéologique des " préfromages » est identique à celui des " rétentats ». Pour des gradients de vitesse inférieurs à $300 \mathrm{~s}^{-1}$, les courbes sont caractéristiques d'une viscosité de structure. Pour des gradients de vitesse supérieurs à $300 \mathrm{~s}^{-1}$, les " préfromages » présentent un comportement newtonien. 
Comme observé au niveau des « rétentats », l'élévation de la température de $2^{\circ} \mathrm{C}$ à $30^{\circ} \mathrm{C}$ réduit fortement la viscosité des " préfromages ». A un gradient de vitesse moyen $437,4 \mathrm{~s}^{-1}$, les valeurs des viscosités des "préfromages " $\mathrm{P}_{1}$ et $\mathrm{P}_{2}$ mesurées à $30^{\circ} \mathrm{C}$ ne représentent respectivement que 40 p. 100 et 10 p. 100 de celles mesurées à $2^{\circ} \mathrm{C}$.

A un gradient de vitesse moyen $\left(437,4 \mathrm{~s}^{-1}\right)$, l'addition de crème au « rétentat " $R_{1}$ provoque une augmentation de la viscosité de l'ordre de 20 p. 100 à $30^{\circ} \mathrm{C}$ et de 28 p. 100 à $2^{\circ} \mathrm{C}$; par contre, l'addition de crème au "rétentat " $\mathrm{R}_{2}$ conduit à une diminution de la viscosité de 21 p. 100 à $30^{\circ} \mathrm{C}$ et de 41 p. 100 à $2^{\circ} \mathrm{C}$. Ce phénomène peut être, en partie, expliqué par l'étude comparée des figures 2,3 et 9 . En effet, au gradient de vitesse $437,4 \mathrm{~s}^{-1}$, la viscosité de la crème qui a servi à la préparation des « préfromages » est élevée par rapport à celle du "rétentat " $\mathrm{R}_{1}\left(115 \mathrm{cP}\right.$ contre $6,5 \mathrm{cP}$ à $\left.2^{\circ} \mathrm{C}\right)$, mais elle est relativement faible par rapport à celle du « rétentat » $\mathrm{R}_{2}$ (115 cP contre $165 \mathrm{cP}$ à $2^{\circ} \mathrm{C}$ ).

Ainsi, la viscosité des " préfromages liquides " dépend principalement de leur teneur en M.G. et de leur teneur en M.A.T. et de la viscosité de la crème utilisée ; on sait aussi que les conditions de préparation et de conservation peuvent conduire à d'importantes variations de viscosité de la crème (Prentice et Chapman, 1969).

\section{DISCUSSION}

Les résultats obtenus au cours de cette étude montrent que les " rétentats " d'ultrafiltration et les " préfromages ", pour des gradients de vitesse faibles, ne sont pas des liquides newtoniens.

Comme le lait condensé (Randhahn, 1973), ils présentent une viscosité de structure d'autant plus importante que la teneur en matière sèche est plus élevée et que la température est plus basse. Pour se rapprocher des conditions de circulation des " rétentats " dans les appareils d'ultrafiltration semi-industriels et industriels, où le gradient de vitesse atteint des valeurs supérieures à $1000 \mathrm{~s}^{-1}$, les mesures de tension de cisaillement ont été réalisées, dans notre étude, à un gradient de vitesse élevé $\left(437,4 \mathrm{~s}^{-1}\right)$ pour lequel les "rétentats" sont des liquides presque newtoniens.

L'accroissement très important de viscosité du "rétentat » que nous avons observé au cours du traitement du lait par ultrafiltration confirme les résultats obtenus par Bourat et Roget (1972). Pour une teneur en substance sèche identique, un " rétentat " a une viscosité plus élevée qu'un lait condensé, ce qui met bien en relief le rôle prépondérant joué par les protéines dans la viscosité du lait et de ses dérivés. 
Bien que peu d'études aient porté sur ce sujet, on peut penser que l'élévation de la teneur en protéines dans le lait concentré et le "rétentat " favorise les interactions entre les micelles de phosphocaséinate de calcium, ce qui aurait pour conséquence une augmentation de la viscosité. Hostettler et Imhof (1951) ont montré que, dans le lait condensé, la taille des micelles était supérieure à celle des micelles du lait original. Plus récemment, Kalab et Harwalkar (1974) ont observé la formation de "ponts ", probablement de nature protéique, entre les micelles, dans les gels obtenus par chauffage de lait écrémé concentré par évaporation ( 40 à $50 \mathrm{~g}$ de substance sèche p. $100 \mathrm{~g}$ de produit).

D'autres observations faites au cours de cette étude inclinent à penser que les éléments constitutifs de ces ponts pourraient être des protéines. En effet, nous avons constaté qu'au cours de la conservation à $2^{\circ} \mathrm{C}$, la viscosité des "rétentats » d'ultrafiltration, préalablement additionnés d'eau oxygénée, s'élevait plus rapidement que la viscosité des "rétentats " n'ayant pas fait l'objet d'une telle addition, ce qui favorisait la formation d'une structure de gel.

Il est probable que l'action oxydante de l'eau oxygénée a pour résultat la création de ponts disulfures entre les micelles de caséine et/ou les protéines du lactosérum riches en acides aminés soufrés.

Il résulte de ces observations qu'il faut éviter l'addition de cet antiseptique aux lactosérum que l'on veut ensuite traiter par ultrafiltration.

En outre, dans le cas d'addition d'eau oxygénée il est nécessaire d'effectuer les mesures de viscosité aussitôt après l'addition de ce produit pour limiter l'influence de son action oxydante sur les propriétés rhéologiques des "rétentats".

Selon toute vraisemblance, les propriétés rhéologiques des " rétentats " d'ultrafiltration et des " préfromages » qui en sont issus dépendent de facteurs tels que le nombre par unité de volume et la dimension des micelles de phosphocaséinate de calcium et des globules gras, la nature et l'importance des associations intermicellaires et la formation de complexes entre les caséines et les protéines du lactosérum.

Toute modification de structure des micelles ou des globules gras se répercute sur la viscosité (au sens large du terme) des "rétentats » et des " préfromages ». C'est ainsi que nous avons constaté, au cours de cette étude, qu'un abaissement modéré du $\mathrm{pH}$ par fermentation lactique provoque une chute de viscosité. Par ailleurs, nous avons observé qu'une homogénéisation modérée (30 à $50 \mathrm{~kg} / \mathrm{cm}^{2}$ ) des « rétentats » (17 à $18 \mathrm{~g}$ p. $100 \mathrm{~g}$ de M.A.T.) ou des " préfromages " (13 à 14 g p. $100 \mathrm{~g}$ de M.A.T. et 13 à 14 g p. $100 \mathrm{~g}$ de M.G.) modifiait la viscosité de ces produits. Ces observations, si elles étaient confrontées avec les résultats obtenus avec d'autres méthodes d'étude des constituants du lait, notamment la microscopie électronique, pour- 
raient constituer une nouvelle voie d'approche dans la compréhension des mécanismes régissant l'état dans lequel se trouvent les différents constituants du lait en solution. De telles observations ont des conséquences pratiques importantes. En effet, un certain nombre de résultats obtenus tant au laboratoire que dans les usines utilisant la technique M.M.V. (Maubois et al., 1969) tendent à montrer que l'homogénéisation modérée des " rétentats " ou des «préfromages " avant emprésurage améliore la qualité organoleptique des fromages ainsi fabriqués (suppression de la texture "sableuse » dans le fromage jeune).

Dans le but d'accroître les performances des appareillages d'ultrafiltration, il est avantageux de diminuer au maximum l'épaisseur de la couche de polarisation de concentration qui se forme au contact des membranes, pour des " rétentats " ayant des teneurs élevées en protéines.

L'abaissement de la viscosité du « rétentat » permet de réduire l'épaisseur de cette couche de polarisation de concentration, et, de ce fait, d'augmenter le flux de perméat (Merson et Ginnette, 1972).

Grâce aux observations réalisées au cours de cette étude, il est possible de préciser certains paramètres physico-chimiques permettant de réduire la viscosité des « rétentats » de lait :

- Il est avantageux, par exemple, que la circulation des " rétentats " au niveau des membranes se fasse à un gradient de vitesse supérieur à $300 \mathrm{~s}^{-1}$, valeur qui conduit à un comportement quasinewtonien du produit et à une viscosité minimale.

- L'ultrafiltration à $2^{\circ} \mathrm{C}$ permet de limiter les développements bactériens ; toutefois, il est difficile, à cause de la viscosité trop élevée, d'obtenir à cette température des « rétentats » ayant une teneur en M.A.T. supérieure à $10 \mathrm{~g}$ p. $100 \mathrm{~g}$. De ce fait, l'ultrafiltration (Maubois, 1973) doit être réalisée soit à une température constante de $30^{\circ} \mathrm{C}$, soit d'abord à $2^{\circ} \mathrm{C}$ (jusqu'à $10 \mathrm{~g}$ de M.A.T. p. $100 \mathrm{~g}$ de "rétentat»), puis à $30^{\circ} \mathrm{C}$.

Cette élévation de température conduit à une diminution de la viscosité de 65 p. 100 pour un « rétentat » à 10 p. 100 de M.A.T. et de 75 p. 100 pour un " rétentat » à 20 p. 100 de M.A.T.

- Enfin, l'abaissement du pH, résultant de la fermentation lactique, provoque un abaissement de la viscosité des " rétentats » pour une teneur en M.A.T. définie. Toutefois, certains auteurs (Maubois et Goudedranche, 1973 ; Muller et al., 1973) ont observé que le débit d'ultrafiltration diminuait lorsque le $\mathrm{pH}$ du « rétentat " s'abaissait.

Cette étude a mis en évidence l'influence de nombreux paramètres sur la viscosité des " rétentats " et des " préfromages ". Les résultats obtenus conduisent à une meilleure connaissance des propriétés rhéologiques de ces produits et devraient servir de base à la détermination des conditions de leur utilisation à l'échelle industrielle. 


\section{R é s u m é}

Les « rétentats » obtenus par ultrafiltration du lait sur membrane ne sont pas des liquides newtoniens ; ils présentent une viscosité de structure d'autant plus importante que le facteur de concentration en protéines est élevé. Toutefois, la viscosité de ces produits est pratiquement indépendante du gradient de vitesse, pour des valeurs de ce gradient supérieures à $300 \mathrm{~s}^{-1}$.

La concentration sélective des protéines au cours de l'ultrafiltration provoque un accroissement très important de la viscosité des "rétentats ". A $2^{\circ} \mathrm{C}$ et pour un gradient de vitesse de $437,4 \mathrm{~s}^{-1}$, la viscosité des « rétentats " évolue de $10,0 \mathrm{cP}$ à $454 \mathrm{cP}$ lorsque la teneur en matières azotées totales varie de 9,0 à 19,6 g p. $100 \mathrm{~g}$.

Pour une teneur en substance sèche totale identique, les « rétentats » de lait ont une viscosité nettement plus élevée que celle des laits condensés.

Les « préfromages liquides " obtenus par addition de crème aux " rétentats " présentent un comportement rhéologique identique à celui des « rétentats "; toutefois, selon les teneurs en protéines et en matières grasses, la viscosité du " préfromage " peut être soit plus élevée, soit plus faible que celle du « rétentat » correspondant.

L'influence de différents facteurs tels que la modification du $\mathrm{pH}$ d'ultrafiltration et de la température, la conservation au froid, l'action de l'eau oxygénée a été étudiée. A partir des résultats obtenus, des hypothèses sur les modifications de la structure des micelles de caséine sont établies et les paramètres physico-chimiques de mise en œuvre de l'ultrafiltration du lait sur membrane sont précisés.

\section{S u m m a ry}

The retentates obtained by membrane ultrafiltration of milk are not Newtonian liquids. They exhibit a structure viscosity which increases with the protein content. However, the viscosity of these products is nearly independent of shear rate for the values of this parameter higher than $300 \mathrm{~s}^{-1}$.

The selective increase in the protein content during ultrafiltration causes a marked increase of the viscosity of the retentate. An increase of the total nitrogenous matter from 9,0 p. 100 up to 19,6 p. 100 induces a retentate viscosity increase from $10,0 \mathrm{cP}$ up to $454 \mathrm{cP}$ (with a shear rate of $437,4 \mathrm{~s}^{-1}$, at $2^{\circ} \mathrm{C}$ ).

At the same total dry matter content, milk retentates have a higher viscosity than condensed milks. 
The liquid drained " cheesemilks " obtained by adding cream to the retentates exhibit the same rheological behaviour as the retentates. However according to their protein and fat contents the viscosity of the liquid drained " cheesemilks » may be higher or lower than the viscosity of the corresponding retentates.

The influence of different factors such as $\mathrm{pH}$, temperature during ultrafiltration and subsequent storage and the effect of adding hydrogen peroxide has been studied. The structure modification of casein micelles after undergoing the ultrafiltration process and the possibilities of determining the physico-chemical characteristics of milk ultrafiltration are discussed.

\section{Remerciements}

Nous tenons à exprimer nos remerciements à MM. Mocquot (I.N.R.A.) et Glover (N.I.R.D.) pour l'aide et les précieux conseils qu'ils nous ont apportés lors de la rédaction de cette publication.

\section{Bibliographie}

BAUcke (A. G.), SANDERSon (W. B.) (1970). - A study of viscosity increase in concentrated skimmilk. XVIII Int. Dairy Congr., Sydney, 1E, 256.

BEeby (R.) (1966). - Heat induced changes in the viscosity of concentrated skimmilk. XVII Int. Dairy Congr., München, EF, 115-121.

BeEBy (R.), Kumetat (K.) (1959). - Viscosity changes in concentrated skimmilk treated with alkali, urea and calcium complexing agents. I. The importance of the casein micelle. J. Dairy Res., 26, 248-257.

BEEBY (R.), LEE (J. W.) (1959). - Viscosity changes in concentrated skimmilk treated with alkali, urea and calcium complexing agents. II. The influence of concentration, temperature and rate of shear. J. Dairy Res., 26, 258-264.

Behrens (G. C.), Morris (H. A.), Coulter (S. T.), Jenness (R.) (1963). - Effects of various heat treatment on the viscosities of simplified milk systems. J. Dairy Sci., 46 (6), 598.

Blatt (W. F.), Dravid (A.), Michaels (A. S.), Nelsen (L.) (1970). - Solute polarization and cake formation in membrane ultrafiltration : causes, consequences and control techniques. In Membrane Science and Technology. Ed, Flinn J. E., Plenum Press, New-York.

Bourat (G.), Roget (G.) (1972). - Rapport contrat D.G.R.S.T. n ${ }^{\circ}$ 70-7-2345. Délégation Générale à la Recherche Scientifique et Technique, Paris.

BundgaARd (A. G.), Olsen (O. J.), MAdSEN (R. F.) (1972). - Ultrafiltration and hyperfiltration of skimmilk for production of various dairy products. Dairy Ind., 37, (10), 539-546.

Cox (C. P.), Hosking (Z. D.), Posener (L. N.) (1959). - Relations between composition and viscosity of cow's milk. J. Dairy Res., 26, 182-189.

FERnANDEZ MaRTin (F.) (1972). - Influence of temperature and composition on some physical properties of milk and milk concentrates. II. Viscosity. J. Dairy Res., 39, 75-82. 
Hostettler (H.), ImHoF (K.) (1951), - Elektronenoptische Untersuchungen über den Feinbau der Milch. Milchwissenschaft, 6, 351-354 et 400-402.

Kalab (M.), HaRWalkar (V. R.) (1974). - Milk gel structure. II. Relations between firmness and ultrastructure of heat-induced skimmilk gels containing 40-60 p. 100 total solids. J. Dairy Res., 41, 131-135.

Kerherve (L.), Richard (J.) (1971). - Influence des résidus de désinfectants dans le lait sur l'activité de quelques streptocoques lactiques. Revue Laitière Française, 291, 717-726.

Maubois (J. L.), Mocouot (G.), Vassal (L.) (1969). - Procédé de traitement du lait et de sous-produits laitiers. Brevet Français no 2052121.

Maubois (J. L.), Goudedranche (N.) (1973). - Résultats non publiés.

Maubois (J. L.), Mocouot (G.) (1971). - Préparation de fromage à partir de " préfromage liquide " obtenu par ultrafiltration du lait. Le Lait, 508, 495-533.

Maubois (J. L.) (1973). - Application de l'ultrafiltration sur membrane à la préparation de différents fromages. Sciences et Techniques, 5, 11-15.

Merson (R. L.), Ginnette (L. F.) (1972). - Reverse osmosis in the food industry. In Industrial Processing with membranes. Ed. Lacey R.E., Loeb S. WileyInterscience, New-York.

Muller (L. L.), Hayes (J. F.), GRIFfin (A. T.) (1973). - Studies on whey processing by ultrafiltration. Aust. J. Dairy Technol., 28, 70-77.

Prentice (J. H.), Chapman (H.R.) (1969). - Some effects of process treatment on the body of cream. J. Dairy Res., 36, 269-278.

RandHaHn (H.) (1973). - Beitrag zum Fliebwerhalten von Milch und Milchkonzentraten. Milchwissenschaft, 28, (10), 620-628.

SAMEL (R.), Muers (M. M.) (1962). - The age thickening of sweetened condensed milk. I. Rheological properties. II. Effects of temperature and of storage. III. The effect of ions. J. Dairy Res., 29, 249-278.

SzCZESNIAK (A. S.) (1973). - Instrumental methods of texture measurements. In texture measurements of foods. Ed. Kramer A., Szczesniak A.S. D. Reidel Publishing Company, Dordrecht (Holland).

Thiele (D.) (1969). - Bestimmung der Veränderung der Emulsionsstabilität von Rahm, nach Lagerung bei verschiedenen Temperaturen mit Hilfe der Viskositatszunahme im Rotations-viskosimeter. Milchwissenschaft, 24, (11), 657-660.

WhitNAH (C. H.) (1962). - The viscosity of milk in relation to the concentration of major constituents and to seasonal differences in the voluminosity of complexes of sedimentable nitrogen. J. Agric. Fd. Chem., 10, (4), 295-296. 